\title{
Review on Bovine Tuberculosis
}

\author{
Tesfaye Belachew* \\ Department of Assela Regional Animal Health Diagnostic Laboratory, Ethiopia
}

Submission: June 26, 2017; Published: September 06, 2017

*Corresponding author: Tesfaye Belachew, Department of Assela Regional Animal Health Diagnostic Laboratory, Assela, Oromiya, Ethiopia, Tel: +251933864966; Email: teyobeku@gmail.com

\begin{abstract}
Mycobacterium bovis is a zoonotic organism and should be treated as a risk/hazard group III organism with appropriate precautions to prevent human infection occurring. Bovine tuberculosis is an infectious disease caused by M. bovis that affects cattle, other domesticated animals and certain free or captive wildlife species. It is usually characterised by formation of nodular granulomas known as tubercles.

Use the Schimmel needle nozzle which is provided with two interchangeable adaptors and the shorter adaptor for a 3/8" needle which gives the recommended projection of 5/32". When changing the Schimmel needle, screw up the needle nozzle against the 'soft' washer until the needle setting is made tight-the washer compresses and this provides friction to prevent the needle nozzle slackening fit the needle hub properly on its seat to prevent leakage. Screw up the needle nozzle, a slight resistance will be felt due to the friction of the soft washer, continue screwing until an increase of resistance is felt when the needle hub reaches its seat and further tighten to make it leak proof. Do not excessively tighten as this destroys the soft metal hub of the needle and will prevent the syringe from filling correctly.
\end{abstract}

Keywords: Mycobacterium bovis; Zoonotic; Schimmel; Tuberculosis

Abbreviations: PPD: Purified Protein Derivative; TB: Tuberculosis; PCR: Polymerase Chain Reaction; OV: Official Veterinarian; CTS: Cattle Tracing System; VMPL: Veterinary Medicinal Products; IR: Inconclusive Reactor; APHA: Animal Health Protection Agency

\section{Introduction}

Tuberculosis is a serious infection in bovine, usually of the lungs, caused by the bacteria Mycobacterium bovis. This is a serious public health problem in many regions of the world, including Southeast Asia and Africa. A Purified Protein Derivative (PPD) skin test is a test that determines if Bovine suffers from Tuberculosis (TB). When TB infects Animal body, it becomes extra sensitive to certain elements of the bacteria, such as the purified protein derivative. A PPD test checks Bovine body's current sensitivity, which will tell whether or not Bovine have TB [1].

Bovine tuberculosis infection in cattle is usually diagnosed in the live animal on the basis of delayed hypersensitivity reactions. Infection is often subclinical; when present, clinical signs are not specifically distinctive and can include weakness, anorexia, emaciation, dyspnoea, enlargement of lymph nodes, and cough, particularly with advanced tuberculosis. After death, infection is diagnosed by necropsy and histopathological and bacteriological techniques. Rapid nucleic acid methodologies, such as the Polymerase Chain Reaction (PCR), may also be used although these are demanding techniques and should only be used when appropriately validated. Traditional mycobacterial culture remains the gold standard method for routine confirmation of infection [2].

Mycobacterium bovis is a zoonotic organism and should be treated as a risk/hazard group III organism with appropriate precautions to prevent human infection occurring. Bovine tuberculosis is an infectious disease caused by $\mathrm{M}$. bovis that affects cattle, other domesticated animals and certain free or captive wildlife species. It is usually characterised by formation of nodular granulomas known as tubercles. Although commonly defined as a chronic debilitating disease, bovine tuberculosis can occasionally assume a more progressive course. Any body tissue can be affected, but lesions are most frequently observed in the lymph nodes (particularly of the head and thorax), lungs, intestines, liver, spleen, pleura, and peritoneum [3].

Ensure on arrival (prior to contact with any livestock) that the vehicle, protective clothing and footwear are clean and suitable for the task being carried out in order to minimize the risk of transmission of disease between premises and when arranging a visit to a farm, ensure that you meet their individual bio security protocols wherever possible, e.g. freedom from contact with other livestock for a given period. However, 
extreme bio security requests should be refused if it makes the task on farm impossible to achieve. On completion of the task, thoroughly clean and disinfect all protective clothing and footwear before leaving the farm premises or appropriately dispose of offsite. Carry sufficient disinfectant approved under the relevant Diseases of Animals [4].

After testing and before leaving the farm, thoroughly clean and disinfect all equipment that is taken onto a farm and that has been in contact with livestock. In testing cattle, goats, pigs or sheep maintain TB syringes in accordance with the manufacturer's instructions. When using disposable syringes, dispose of them as pharmaceutical waste along with all used needles and part used/opened bottles of tuberculin [5].

\section{Materials and Methods}

\section{Study methodology}

Data collection: Questioner format was developed and all information relating to the study objectives were recorded. Data which was recorded includes type of Animal ID, dairy husbandry system, Body condition, sex, breed, and age.

\section{Skin testing procedures}

TB skin tests are carried out on two days: Day one - injection of avian and bovine tuberculin and day two - reading of test 72 hours later. The complete tuberculin test must be carried out by the same Official Veterinarian (OV) unless there are exceptional circumstances [6]. OVs therefore should not commence a test that they know.

Care to be taken in procedure: If, for whatever reason, the test cannot be completed notify APHA. An OV is responsible for completing a risk assessment regarding their health and safety when conducting on-farm testing and inspection for APHA [7]. APHA will support OVs in any appropriately evidenced decision to withdraw from a test on health and safety grounds. It is not appropriate to cut corners on the test procedure on the grounds of health and safety. If the test cannot be conducted properly for health and safety reasons, it should be abandoned.

Care to be taken with Injection: Cattle must not be moved off the holding between the injection of tuberculin (TT1 date) and the reading of the test (TT2 date). In all cases the same set of Digital caliper, or the same type of Digital caliper calibrated to produce identical readings, must be used on both test days. Refer to records made at the time of injection to ensure correct reading and interpretation of the test (e.g. inadvertent subcutaneous injection, unforeseen skin blemishes etc.).

\section{How to Prepare the TB Syringe}

Assembling: Apply a thin coating of lubricating oil, recommended by the manufacturer, to the plunger and grip the pawls between your fingers, thumb to clear the ratchet teeth and extend the plunger approximately halfway out of the frame [8]. Slacken the sealing ring gland slightly to allow the plunger to enter freely and tighten the sealing ring gland until it just stops against the inner sealing ring ('o' ring), then very slightly tighten to prevent leakage. Screw the barrel onto the frame and grip the pawls and move the plunger up and down several times. Screw on the main nozzle only moderately tight, just sufficient to prevent leakage.

Needles: Use new or sterilized needles for each herd tested and dispose of old ones safely. Change needles immediately if they become contaminated with blood or any other material during TB testing. Use Record or Schimmel needles. The Special Record needle for the McLintock syringe is particularly recommended.

Fitting record needles: Use the special washer with a metal rim provided, do not use the soft rubber washer. The washer is used to prevent leakage and the metal rim prevents the soft interior from spreading and blowing out under pressure.

Fitting schimmel needles: Use the Schimmel needle nozzle which is provided with two interchangeable adaptors and the shorter adaptor for a 3/8" needle which gives the recommended projection of 5/32". When changing the Schimmel needle, screw up the needle nozzle against the 'soft' washer until the needle setting is made tight-the washer compresses and this provides friction to prevent the needle nozzle slackening.fit the needle hub properly on its seat to prevent leakage [9]. Screw up the needle nozzle, a slight resistance will be felt due to the friction of the soft washer, continue screwing until an increase of resistance is felt when the needle hub reaches its seat and further tighten to make it leak proof. Do not excessively tighten as this destroys the soft metal hub of the needle and will prevent the syringe from filling correctly.

Filling the syringe: It is essential to ensure that there is no air left in the syringe after filling, as this can lead to incorrect doses being administered and withdraw the plunger to its fullest extent. Insert the needle through the rubber cap of the relevant tuberculin vial, with the vial inverted (so air bubbles will rise) and ensure that the needle stays under the liquid whilst filling the syringe. Keeping the vial and syringe vertical can help [10]. Injecting air into the vial equivalent to the amount of liquid to be withdrawn may help. As there is little space in some bottles this may have to be done in stages. Retract the plunger slowly to draw liquid into the syringe and remove any air present in the syringe using the methods.

Method to fill the needle: With the syringe held vertically and vial inverted, tap the barrel vigorously with your finger, air bubbles will rise into the conical main nozzle and expel trapped air by moving the plunger two to three increments or until only liquid is being injected back into the vial i.e. no sign of bubbles from the needle. All air has now been expelled from the syringe and maintains the vertical position and slowly withdraws the plunger to its fullest extent, or alternatively to the number of doses required. Withdraw the needle from the vial and the syringe is now correctly filled and ready for use. If a droplet issues from the needle after an injection has been made, this indicates that air is present within the syringe. Repeat the process above 
to remove the air and if the syringe used for avian tuberculin is accidentally filled with bovine tuberculin or vice versa, empty and cleanse the syringe.

\section{Day one testing actions}

Animal identification: All animals to be tested must be identified by the official ear number as required by the relevant Cattle Identification Regulations. Owners are required to ensure all cattle on their holding comply with the regulations for identification (ID) and recording on the Cattle Tracing System (CTS).

Record the official ear tag number or registered name on the testing record [11]. Other management numbers (e.g. freeze brands) can be recorded in the testing record but must not be given as the sole identification. Record all alternative identification marks for any animals where there is doubt regarding the official ear tag number. If an animal with no approved or insufficient method of identification is presented for testing, it must either be, tagged or retagged in accordance with the requirements of the relevant Cattle.

Eligibility for test: Owners are required to present for testing all eligible cattle on the holding appropriate for the type of test being conducted. Any doubts, particularly about the eligible animals not being presented, should be noted on the testing record to ensure any concerns are raised to APHA.

Other veterinary medicinal products: Veterinary Medicinal Products (VMPs) should not be administered at the time of first injection unless for welfare reasons which should be noted on the test chart/report. Where VMPs are administered on day one details must be recorded on the testing record, for entering on to Sam (where necessary).

\section{Preparation of the injection site}

Careful intradermal injection of tuberculins is critical to an accurate and consistent performance of the comparative tuberculin test [12]. This is because the sensitivity of the test varies in different areas of the neck and decreases towards the shoulder. Ensure there are no skin blemishes or other pathological conditions present at the selected sites which might interfere with the skin measurement or the test. In these cases, the other side of the neck can be used. Both sites shall normally be in the border of the anterior and middle third of one side of the neck.

For Inconclusive Reactor (IR) retests, the injections should be made (if possible) on the opposite side of the neck to that used at the previous test. The upper site (avian tuberculin) should be, in the adult bovine, at least $10 \mathrm{~cm}$ below the crest, and the lower site (bovine tuberculin) should be approximately $12.5 \mathrm{~cm}$ from the other, on a line roughly parallel with the line of the shoulder, as in the picture below. In order to maximize test sensitivity, injection sites should be as close as possible to those indicated [13].
Injection sites shall be clipped and cleansed to physically remove debris. Clip two small areas of the neck for the intradermal injection of tuberculin ensuring that both injection sites are clearly visible and free of hair and blemishes. In young cattle, when there is not enough room to separate the sites sufficiently on one side of the neck, make one injection on each side of the neck at identical sites in the centre of the middle third of the neck. Site the avian injection on the left hand side of the neck and the bovine on the right. For calves, this is considered standard practice.

Measure skin thickness: Raise a fold of skin at each test site, measure the thickness of the fold with the Digital callipers and record the measurements in millimeters in the testing record. The upper site should be used for the avian tuberculin (or the left hand side of the neck for young animals). In the event that this injection site protocol is inadvertently reversed, ensure that this is clearly stated in your notes.

Injecting tuberculin: The dose of tuberculin shall then be injected by a method that ensures that the tuberculin is delivered intradermally. Inject the avian tuberculin into the upper site for the (or the left hand side of the neck for young animals) using the syringe with the red button. Inject the bovine tuberculin into the lower site (or the right hand side of the neck for young animals) using the syringe with the blue button. Avoid subcutaneous injection as this could potentially give rise to a false test result and desensitization of the site for a variable period [14]. Check that a palpable nodule is present in the skin at each injection site. This indicates that the injection has been correctly made. This should usually be about the size of a pea. If a palpable nodule is not present and it is thought that the tuberculin may have been injected subcutaneously, inject again using sites on the other side of the neck. Record this action in the testing record.

Clinical inspections and examinations: Carry out a visual inspection of all cattle tested on day one and day two. This is to identify any animals which are showing clinical signs of tuberculosis, skin tuberculosis and any notifiable disease of cattle (e.g. Foot and Mouth Disease etc.). The purpose of this is to assist with the identification of allergic animals and animals not fit for human consumption $[15,16]$. Where clinical signs consistent with bovine tuberculosis are seen a clinical examination must be carried out and findings recorded on the remarks column or in appropriate place on the testing record.

\section{Result}

Table 1

\begin{tabular}{|c|c|c|c|}
\hline Number of & TB positive & Tb suspected & Total \\
\hline $\begin{array}{c}\text { Animal } \\
\text { examined }\end{array}$ & $\begin{array}{c}\text { Positive No. } \\
(\%)\end{array}$ & $\begin{array}{c}\text { Suspected No. } \\
(\%)\end{array}$ & \\
\hline 37 & $1(2.7 \%)$ & $7(18.9 \%)$ & $8(21.6 \%)$ \\
\hline
\end{tabular}

\section{References}

1. Angus RD (1978) Production of reference PPD tuberculins for veterinary use in the United States. J Biol Stand 6(3): 221-227. 
2. Cousins DV, Francis BR, Gow BL (1989) Advantages of a new agar medium in the primary isolation of Mycobacterium bovis. Vet Microbiol 20(1): 89-95.

3. Clifton-HRS, Wilesmith JW (1991) Tuberculosis in deer: a review. Vet Rec 129(1): 5-12.

4. Buddle BM, Ryan TJ, Pollock JM, Andersen P, de Lisle GW (2001) Use of ESAT- 6 in the interferon-gamma test for diagnosis of bovine tuberculosis following skin testing. Vet Microbiol 80(1): 37-46.

5. Bengis RG, Kriek NP, Keet DF, Raath JP, de Vos V, et al. (1996) An outbreak of tuberculosis in a free-living African buffalo (Syncerus cafferSparrman) population in the Kruger National Park: a preliminary report. Onderstepoort J Vet Res 63(1): 15-18.

6. Animal Health Division (New Zealand) (1986) Possum research and cattle tuberculosis. Surveillance 13: 18-38.

7. Aranaz A, Cousins D, Mateos A, Domínguez L (2003) Elevation of Mycobacterium tuberculosis subsp. caprae Aranaz et al. 1999 to species rank as Mycobacterium caprae comb. nov., sp. nov. Int J Syst Evol Microbiol 53(Pt 6): 1785-1789.

8. Coad M, Hewinson RG, Clifford D, Vordermeier HM, Whelan AO (2007) Influence of skin testing and blood storage on interferon-gamma production in cattle affected naturally with Mycobacterium bovis. Vet Rec 160(19): 660-662.

9. Cockle PJ, Gordon SV, Hewinson RG, Vordermeier HM (2006) Field evaluation of a novel differential diagnostic reagent for detection of

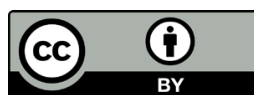

This work is licensed under Creative Commons Attribution 4.0 Licens DOI: 10.19080/JDVS.2017.03.555611
Mycobacterium bovis in cattle. Clin Vaccine Immunol 13 (10): 11191124

10. Cornerl LA (2006) The role of wild animal populations in the epidemiology of tuberculosis in domestic animals: how to assess the risk. Vet Microbiol 112(2-4): 303-312.

11. Cousins DV, Florisson N (2005) A review of tests available for use in the diagnosis of tuberculosis in non-bovine species. Rev sci tech Off Int Epiz 24(3):1039-1059.

12. Cousins DV (2001) Mycobacterium bovis infection and control in domestic livestock. Rev Sci Tech 20(1): 71-85.

13. Griffin JFT, Cross JP, Chinn DN, Rogers CR, Buchan GS (1994) Diagnosis of tuberculosis due to $\mathrm{M}$. bovis in New Zealand red deer (Cervus elaphus) using a composite blood test (BTB) and antibody (ELISA) assays. NZ Vet J 42:173-179.

14. Haagsma J, O reilly LM, Dobbelaar R, Murphy TM (1984) A comparison of the relative potencies of various bovine PPD tuberculins in naturally infected tuberculous cattle. J Biol Stand 10(4): 273-284.

15. Schneider W, Dobbelaer R, Dam A, Jorgensen JB, Gayot G, et al. (1979) Collaborative assay of EEC standards for bovine tuberculins. J Biol Stand 7: 53.

16. Wilesmith JW (1991) Epidemiological methods for investigating wild animal reservoirs of animal disease. Rev Sci Tech 10(1): 205-214.

\section{Your next submission with Juniper Publishers} will reach you the below assets

- Quality Editorial service

- Swift Peer Review

- Reprints availability

- E-prints ServiceManuscript Podcast for convenient understanding

- Global attainment for your research

- Manuscript accessibility in different formats ( Pdf, E-pub, Full Text, Audio)

- Unceasing customer service

Track the below URL for one-step submission https://juniperpublishers.com/online-submission.php 